\title{
Las tecnologías hi-tech en los grados en Periodismo. Planes de estudios, formación de los periodistas y propuestas de inserción curricular Hi-tech technologies in the degrees in Journalism. Curricula, training of journalists and proposals for curricular insertion
}

María José Ufarte Ruiz Universidad de Castilla-La Mancha

Luis Mauricio Calvo Rubio Universidad de Castilla-La Mancha

Francisco José Murcia Verdú Universidad de Castilla-La Mancha

\section{Referencia de este artículo}

Ufarte Ruiz, María José; Calvo Rubio, Luis Mauricio y Murcia Verdú, Francisco José (2020). Las tecnologías hi-tech en los grados en Periodismo. Planes de estudios, formación de los periodistas y propuestas de inserción curricular. En adComunica. Revista Científica del Estrategias, Tendencias e Innovación en Comunicación, $\mathrm{n}^{0}$ 20, Castellón: Universitat Jaume I, 43-66. DOI: http://dx.doi.org/10.6035/21740992.2020.20.3.

\section{Palabras clave}

Alta tecnología; planes de estudio; grado; formación; inserción curricular; periodismo.

\section{Keywords}

High technology; Study Plans; Degree; Training; Curricular Insertion; Journalism. 


\title{
Resumen
}

El objetivo de esta investigación es triple: identificar, desde una perspectiva exploratoria, la oferta formativa relacionada con las altas tecnologías en los grados en Periodismo de las universidades españolas, conocer si los periodistas que trabajan en los medios de comunicación cuentan con competencias y habilidades específicas para utilizar estas herramientas en la creación de contenidos y proponer diferentes materias docentes para la inserción curricular del periodismo hi-tech. Para alcanzar estos propósitos se ha empleado una metodología mixta, con procedimiento de tipo cualitativo, como el análisis de contenido aplicado a las 768 asignaturas que configuran los 17 planes de estudios de grado que son objeto de esta investigación, y cuantitativo, como el cuestionario online suministrado a profesionales del sector $(\mathrm{N}=131)$ con el fin de saber si poseen formación especializada para utilizar las altas tecnologías en los procesos comunicativos. Los resultados revelan que la enseñanza-aprendizaje de las tecnologías emergentes y disruptivas de la información empieza a identificarse en los grados en Periodismo de las universidades públicas españolas. En el terreno laboral, los profesionales también comienzan a formase sobre cómo aplicar la inteligencia artificial, los drones, los móviles y las técnicas inmersivas a la creación de contenidos. Sin embargo, la oferta formativa sobre esta disciplina es aún incipiente, por lo que se proponen diferentes materias en las que puede englobarse la enseñanza-aprendizaje del periodismo hi-tech en los grados en Periodismo.

\begin{abstract}
The objective of this research is threefold. First, the training offer related to high technologies in degrees in Journalism at Spanish universities is identified. Second, it's intended to know whether journalists who work in the media have competences and skills specific to use these tools in the creation of content. Finally, different teaching subjects for the curricular insertion of hi-tech journalism have proposed. To achieve these purposes, a mixed methodology has been used, with a qualitative type procedure, such as content analysis applied to the 768 subjects that make up the 17 undergraduate study plans that are the subject of this research; and quantitative, such as the online questionnaire provided to professionals in the sector $(\mathrm{N}=131)$ in order to know if they have specialized training to use high technology in communication processes. The results reveal that the teaching-learning of emerging and disruptive information technologies is beginning to be identified in the degrees in Journalism of Spanish public universities. In the workplace, professionals also begin training on how to apply artificial intelligence, drones, mobiles and immersive techniques to content creation. However, the training offer on this discipline is still in its infancy, so different subjects are proposed in which the teaching-learning of hi-tech journalism can be included in the degrees in Journalism.
\end{abstract}




\section{Introducción}

La evolución tecnológica ha cambiado radicalmente el periodismo (Pavlik, 2001). La digitalización de los procesos informativos ha transformado las rutinas profesionales (Micó y Pérez, 2015) y ha abierto nuevas vías para la producción (Hermida y Thurman, 2008) y el consumo de contenidos (Casero-Ripollés, 2012; Westlund, 2013). La mediamorfosis mediática (Fidler, 1997) invita a revisar la ecología de los medios propuesta por McLuhan (1964) y Postman (1970) para adaptarla al nuevo escenario comunicativo, donde una tecnología supera a la anterior sin apenas tiempo para su aprovechamiento (Lozano, 2017) y sin que se atisbe un período de estabilidad social y profesional en los medios de comunicación (Salaverría, 2019).

Esta transformación tecnológica exige innovación periodística (López y Aramburú, 2016; Paulussen, 2016). Del proceso de cambios acaecidos, ya despuntan nuevas líneas de trabajo que determinarán la evolución del periodismo en los próximos años (Salaverría, 2014). Entre otras, estas vías van desde los laboratorios de innovación (García-Avilés, 2018; López; Ufarte, 2016) hasta el periodismo hi-tech o de alta tecnología (Murcia y Ufarte, 2019), que se caracteriza por el uso de tecnologías disruptivas (Christensen, 1999) en el ámbito periodístico. En concreto, Fernández (2018a) y López (2016) señalan la utilización de inteligencia artificial, drones, smartphones y técnicas inmersivas, que expresan todo su potencial con tecnologías y equipos de realidad virtual (Domínguez, 2015).

Estas técnicas de última generación ofrecen nuevas posibilidades para la creación de contenidos periodísticos y ya forman parte de la radiografía actual de los medios de comunicación, permitiendo nuevas coberturas, una mejor relación con las audiencias y una respuesta más ágil y eficiente a noticias de última hora (Lecompte, 2015).

En el campo de los drones, la CNN, The Daily Dot, Manchester Evening, BCC, Rusia Today, CBS y TF1, entre otros, han utilizado los vehículos aéreos no tripulados con fines periodísticos para obtener imágenes desde el aire de difícil cobertura con perspectiva, rapidez, movilidad y mayor seguridad para los periodistas (Aguado, 2019; Barreno, 2019). Los medios en España no han adoptado esta tecnología y prefieren, por el momento, subcontratar los servicios. Así lo han hecho, por ejemplo, Mediaset, Radio Televisión Pública de Andalucía (RTVA) y Radiotelevisión Española (RTVE), entre otras, debido principalmente a la falta de regulación y al elevado coste de estos sistemas (Fernández, 2018b).

En el terreno de la inteligencia artificial, entendida como la aplicación de bots y algoritmos para la escritura automática de textos informativos (Barrat, 2013; Bunz, 2010), Túñez, Toural y Cacheiro (2018) han contabilizado a nivel mundial un total de 16 medios periodísticos, 13 agencias de noticias y 21 empresas que recurren a la automatización. En el ámbito nacional, Ufarte y Manfredi (2019) destacan el caso de Narrativa Inteligencia Artificial, que es la única empresa del 
país que genera noticias redactadas a partir de inteligencia artificial a diferentes medios de comunicación, como Sport, 20 Minutos, El Periódico, El Confidencial, El Español, El Independiente, El Heraldo y La Información. También la Agencia EFE se ha unido a esta tendencia.

Los smartphones y tabletas también han cobrado protagonismo en el sector al permitir una mayor interactividad, inmediatez y multimedialidad a la hora de redactar editar y distribuir la información. Es lo que se conoce como periodismo móvil o MOJO, de Mobile Journalism (López García et. al, 2019), una tendencia que ya practican la BBC, The Times, The New York Times, Reuters, La Repubblica Sera, O Globo y Leman Bleu, entre otras (Larrondo y Teixeira, 2016). A nivel nacional, destacan las coberturas de RTVE sobre las iniciativas turísticas para combatir la España vaciada, la Cumbre del Clima de Madrid o las reacciones de los últimos visitantes a la tumba de Franco.

Pero es importante que las historias vayan más allá y permitan viajar de algún modo al lugar de los hechos. Es lo que aporta el periodismo inmersivo, el vinculado a contenidos que, basándose en el uso de entornos de realidad virtual e interactividad, buscan proporcionar al espectador una perspectiva en primera persona de los hechos narrados (De la Peña et al., 2010). A nivel internacional, despuntan las experiencias de The New York Times, CNN, BBC, Usa Today, ABC (EEUU), The Economist, The Guardian, Wall Street Journal, AP, DW, RT, Euronews, Nat Geo, Discovery, Al Jazeera, Huffigton Post, FOX Sports, Sport Illusrated y LIFE, entre otras (Herranz, Sidorenko y Cantero, 2019). En España, destacan las coberturas inmersivas de RTVE, El País, Antena 3, El Confidencial, El Español, Diario de Navarra, Diario de Sevilla, Diario Montañés, Faro de Vigo, 8 Zamora, El Diario Conquense, Castilla-La Mancha Media (CMM), Radio Televisión de Castilla y León, Canal Sur, Televisió de Catalunya (TV3), Televisión de Galicia (TVG), Radiotelevisió de les Illes Balears (IB3) y Canal Extremadura, entre otros (Cantero, Calvo y Benedicto, 2020).

La utilización de estas herramientas caracteriza una parte de los perfiles periodísticos más demandados en el sector (Ufarte, 2019), debido a que los periodistas recurren cada vez más a las aplicaciones avanzadas para afrontar sus desafíos profesionales. Es decir, precisan con mayor asiduidad de herramientas más sofisticadas para la búsqueda de datos, para la elaboración de las piezas informativas, para su difusión y para comunicarse con los usuarios, en especial con las audiencias más activas (Newman, 2020).

Su uso, por tanto, continuará en los próximos años (Eudes, 2014), pero el ejercicio del periodismo de alta tecnología requiere del manejo de herramientas que el periodista deberá conocer (Anderson, 2012). Por eso, desde el punto de vista profesional, el primer desafío es preparar a profesionales multiplataforma y multimedia (Salaverría, García-Avilés y Masip, 2007) para trabajar en nuevos entornos 
(Tejedor-Calvo, Corpus y Lozano, 2011), ya que la mejor forma de atraer al público es hacer un producto diferente, lo que implica la formación en nuevos lenguajes (Canavilhas, 2015) y que entiendan la red en toda su dimensión (Van der Haak, Park y Castells, 2012).

En general, los periodistas han sido autodidactas en el uso de las tecnologías digitales, pero, poco a poco, la innovación también está transformando profundamente las universidades (Flores Vivar, 2019). En este contexto, es necesaria la preparación universitaria en competencias transversales y específicas en temáticas relacionadas con las tecnologías emergentes y disruptivas de la información, aunque no es sencillo introducir asignaturas y enfoques innovadores, a pesar de las novedades de la industria, en general, y del oficio, en particular (Hewett, 2015). Algunos países son conscientes de las oportunidades que conlleva esta nueva vía de especialización. Francia, por ejemplo, triplicará en los próximos años el número de profesionales formados en inteligencia artificial, tanto a nivel universitario (grado, máster, doctorado) como de formación profesional, y Alemania creará cien nuevas cátedras orientadas a formar a profesionales en automatización (Villani, 2018).

En este contexto, el objetivo de esta investigación es triple: 1) identificar, desde una perspectiva exploratoria, la oferta formativa relacionada con las altas tecnologías en los grados en Periodismo de las universidades públicas españolas; 2) conocer si los periodistas que trabajan en los medios de comunicación cuentan con competencias y habilidades específicas para utilizar estas herramientas en la creación de contenidos y 3) proponer diferentes materias para la inserción curricular del periodismo hi-tech. El trabajo recurre a la metodología cualitativa y cuantitativa y parte de las siguientes hipótesis:

- H1. La enseñanza-aprendizaje de las tecnologías emergentes y disruptivas de la información, que generalmente tienen como plataformas a internet y la telefonía móvil, empieza a identificarse en los grados en Periodismo de las universidades públicas españolas.

- H2. El sector periodístico es consciente de que debe adaptarse a los nuevos tiempos y aplicar estas herramientas a los métodos tradicionales de generación de noticias, por lo que los periodistas ya empiezan a formarse sobre las herramientas hi-tech, a pesar de que un buen número las empresas informativas todavía carecen de estas tecnologías emergentes.

- H3. Existen diferentes disciplinas en las que puede englobarse la enseñanzaaprendizaje del periodismo de alta tecnología con el fin de que la universidad se adapte a los cambios que está sufriendo la industria periodística y dé cabida a nuevos contenidos con docentes formados en competencias digitales. 


\section{Metodología}

Esta investigación se articula a partir de los siguientes objetivos: 1) Identificar, desde una perspectiva exploratoria, la oferta formativa relacionada con las tecnologías emergentes y disruptivas de la información en los grados en Periodismo de las universidades españolas. 2) Conocer las competencias y habilidades que tienen los periodistas para utilizar las altas tecnologías en la creación de contenidos periodísticos. 3) Proponer diferentes disciplinas en las que puede englobarse la enseñanzaaprendizaje de las altas tecnologías en los procesos comunicativos. Alcanzar estos propósitos es una cuestión importante para la supervivencia de los medios en un contexto marcado por el cambio constante en la tecnología, los comportamientos de la audiencia, las estrategias de la competencia y los modelos de negocio.

El trabajo de campo se ha desarrollado en cuatro fases, en las que se ha producido una triangulación metodológica (Gaitán y Piñuel, 1998), que según Gómez-Diago (2010) y Soler y Enrique (2012), consiste en contrastar información entre distintas fuentes para obtener una contextualización suficiente de los fenómenos estudiados.

En primer lugar, se ha realizado una revisión de la literatura científica, que forma parte de la investigación secundaria (Codina, 2017), y que ha permitido conocer las principales aportaciones al estado de la cuestión. En segundo lugar, se han analizado los planes de estudio de las enseñanzas conducentes a la obtención del título universitario oficial de graduado en Periodismo de 17 centros nacionales (Tabla 1). Esta muestra es el resultado de la intersección entre las 10 universidades con mayor número de plazas ofertadas en el curso 2018-2019 y con mayor nota de corte en Periodismo en ese mismo año académico, según los datos del Ministerio de Educación, Cultura y Deporte acotados a universidades públicas. Aunque la muestra queda lejos de los 39 grados de Periodismo que forman parte del Registro de Universidades, Centros y Títulos (RUCT) del Ministerio de Ciencia, Innovación y Universidades -20 impartidos en centros públicos y 19 en privados-, se considera que es representativa para un estudio de estas características debido a los criterios de selección empleados.

Delimitada la muestra de estudio, se ha elaborado una ficha de análisis para obtener de cada centro datos referentes a las asignaturas dedicadas total o parcialmente a las tecnologías emergentes y disruptivas de la información. De esta manera, se han analizado las competencias y el contenido de todas las materias que configuran los 17 planes de estudio para localizar menciones a temas relacionados con el objeto de estudio. También se ha recabado información sobre el curso en el que se imparte, los ECTS asignados y la modalidad académica, diferenciando si son asignaturas de formación básica (las que establece el Ministerio de Educación pertenecientes a las áreas de conocimiento afines a los grados), obligatorias (las establece cada universidad) o asignaturas optativas (las elige el alumno, a propuesta de la universidad, para completar su formación) (Sánchez, 2013). Este estudio se 
Tabla 1. Muestra de universidades

\begin{tabular}{|l|c|c|c|}
\hline Universidad & Tipo / Centro & Oferta de plazas & Nota de corte \\
\hline Complutense de Madrid & Pública / Propio & 620 & 7,848 \\
\hline Autónoma de Barcelona & Pública / Propio & 280 & 9,462 \\
\hline Universidad de Sevilla & Pública / Propio & 280 & 8,023 \\
\hline País Vasco & Pública / Propio & 210 & 7,46 \\
\hline Rey Juan Carlos & Pública / Propio & 160 & 6,995 \\
\hline Málaga & Pública / Propio & 130 & 8,54 \\
\hline Valladolid & Pública / Propio & 120 & 5,487 \\
\hline EUSA-Sevilla & Pública / Adscrito & 120 & 5 \\
\hline Carlos III de Madrid & Pública / Propio & 105 & 10,282 \\
\hline Castilla-La Mancha & Pública / Propio & 99 & 5,056 \\
\hline Jaume I de Castellón & Pública / Propio & 90 & 9,07 \\
\hline Pompeu Fabra & Pública / Propio & 80 & 11,762 \\
\hline Valencia & Pública / Propio & 80 & 10,76 \\
\hline Santiago de Compostela & Pública / Propio & 75 & 9,55 \\
\hline Murcia & Pública / Propio & 70 & 8,526 \\
\hline Zaragoza & Pública / Propio & 60 & 10,472 \\
\hline Rovira i Virgili & Pública / Propio & 40 & 836 \\
\hline
\end{tabular}

Fuente: Ministerio de Educación, Cultura y Deporte.

ha efectuado en base al análisis de contenido web (Herring, 2010), que complementa al tradicional (Bardin, 1977; Krippendorf, 2004).

En tercer lugar, se ha distribuido un cuestionario online de preguntas cerradas entre 131 periodistas, con el fin de conocer sus competencias y habilidades sobre las altas tecnologías en la creación de contenidos periodísticos. La muestra está compuesta por un $55 \%$ de mujeres y un $45 \%$ de hombres de edades comprendidas entre los 20 y los 60 años, aunque la mayoría se encuentra en una franja entre 31 y 40 años (45,7\%). Del total, un 41,9\% trabaja en prensa, un 27,9\%, en gabinetes de comunicación, un $10,9 \%$, en radio y un $8,5 \%$ en televisión. El 10,8\% restante desempeña sus funciones en medios digitales, revistas y editoriales. Para su selección, se ha aplicado la técnica del muestreo no probabilístico por conveniencia (Perlado, Papí y Bergaz, 2019). El número de encuestados está en sintonía con el 
de otras investigaciones similares, como la de Moreiro González et. al, (2008), que cuenta con 118 encuestados, o la de Bermejo, Campos y Sánchez (2017), que realiza una encuesta a 96 profesionales del sector. A la muestra se le ha explicado qué se considera por alta tecnología (o denominaciones similares) y se le ha preguntado, entre otras cuestiones, sobre los siguientes temas: ¿el medio para el que trabaja apuesta por las tecnologías emergentes para la creación de contenidos?, ¿ha recibido algún tipo de formación para utilizar las altas tecnologías (hi-tech) en los procesos comunicativos?, ¿ha utilizado alguna vez técnicas avanzadas de co-creación de contenidos?, ¿cree que el uso de tecnologías de última generación ofrece ventajas para la profesión periodística?, ¿considera que la alta tecnología aplicada al periodismo ofrece nuevas posibilidades para la creación de contenidos?

A partir de los resultados obtenidos en las anteriores etapas de la investigación, se realiza, en cuarto lugar, una propuesta de diferentes disciplinas en las que puede englobarse la enseñanza-aprendizaje de las altas tecnologías en los procesos comunicativos. Al abogar por esta oferta se defiende la formación de periodistas con habilidades tecnológicas adaptadas al actual ecosistema mediático, en línea con los planteamientos de Caminero y Sánchez (2018) y Gómez-Calderón, Roses y García-Borrego (2017), entre otros.

Con esta combinación metodológica de técnicas cualitativas y cuantitativas se visualiza el problema de investigación desde diferentes ángulos, se evitan sesgos y fallas metodológicas para optimizar la validez y la consistencia de los hallazgos (Okuda y Gómez, 2005). Siguiendo las investigaciones de Yin (1981) sobre estudios de casos, este trabajo no pretende realizar una generalización estadística, sino analítica, debido a que busca un patrón de comportamientos y no una enumeración de frecuencias de la muestra (Villareal y Landeta, 2010). Este estudio es oportuno por su valor descriptivo al analizar en detalle el panorama formativo sobre las tecnologías emergentes y disruptivas de la información en Periodismo y su incidencia en la educación superior universitaria en un momento en el que el ritmo acelerado de los cambios económicos, sociales y tecnológicos ha acentuado la importancia de la innovación para lograr una ventaja competitiva en el sector periodístico.

\section{Resultados}

\subsection{Oferta académica en Periodismo}

El periodismo hi-tech es un contenido que empieza a identificarse en los grados en Periodismo de las universidades españolas (Tabla 2). Así se demuestra tras analizar 768 guías docentes correspondientes a los 17 planes de estudios de grado que son objeto de esta investigación, que ha dejado al margen las asignaturas correspondientes a los trabajos de fin de grado y a las prácticas, así como aquellas 
que no ofrecen de manera online su guía docente. Este último caso representa menos del $1 \%$ de la totalidad.

La oferta curricular sobre inteligencia artificial está presente en los planes de estudios de las facultades de Ciencias de la Comunicación de la Universidad de Málaga y de la Autónoma de Barcelona. En el caso de la institución andaluza es a través de la asignatura optativa Información en Internet ( $4^{\circ}$ curso, 6 ECTS), que cuenta con un contenido específico de periodismo robot o la automatización de la profesión. Por su parte, el centro catalán aborda el uso de bots y algoritmos en la redacción de noticias en las materias obligatorias Fundamentos tecnológicos del periodismo $\left(1^{\mathrm{o}}\right.$ curso, 6 ETC) y Políticas de comunicación ( $3^{\circ}$ curso del plan extinción, 6 ECTS).

Respecto al periodismo dron, no existe ninguna materia que refiera el uso de los vehículos aéreos no tripulados en el ámbito periodístico de manera monográfica, como área de especialización o en las competencias a adquirir.

Por el contrario, el periodismo móvil está presente en más de la mitad de los planes de estudios analizados. La Autónoma de Barcelona lo imparte en dos asignaturas obligatorias: Fundamentos tecnológicos del periodismo ( $1^{\circ}$ curso, 6 ECTS) y Producción y expresión periodística en multimedia e interactivos $\left(2^{\circ}\right.$ curso del plan en extinción, 6 ECTS), mientras que la Facultad de Comunicación de Sevilla lo hace en Producción periodística (obligatoria, $2^{\circ}$ curso, 6 ECTS) y Periodismo multimedia y diseño gráfico digital (optativa, $3^{\circ}$ curso, 6 ECTS). En esta misma ciudad, el centro adscrito EUSA cuenta en su oferta curricular con dos materias que abordan el periodismo móvil: Cyberculture (obligatoria para los que opten por la enseñanza en inglés, $4^{\circ}$ curso, 6 ECTS) y Periodismo multimedia y diseño gráfico digital -y su versión en inglés Multimedia journalism \& digital graphic design- (optativas, $3^{\circ}$ curso, 6 ECTS). En Andalucía, la Facultad de Málaga también ofrece la posibilidad de adentrarse en esta disciplina en la asignatura optativa Fotoperiodismo e imagen digital, que se imparte en $4^{\circ}$ curso (6 ECTS). Por su parte, la Facultad de Ciencias Sociales y de la Comunicación del País Vasco estudia los dispositivos móviles con fines periodísticos en Tecnología del periodismo (obligatoria, $2^{\circ}$ curso, 6 ECTS) y Periodismo social y participación en internet (optativa, $3^{\circ}$ curso, 6 ECTS). Y la Facultad de Ciencias da Comunicación de la Universidad de Santiago de Compostela cuenta con la asignatura obligatoria Multimedia: teoría, técnica y aplicaciones (6 ECTS), en la que se imparten diferentes nociones del periodismo móvil, como la adaptación de los contenidos a los nuevos soportes digitales, las redes sociales y uso del vídeo y la audiencia social. De igual modo, la Facultad de Comunicación y Documentación de Murcia oferta una asignatura anual en tercer curso llamada Tecnologías de la producción informativa que aborda el uso del móvil con fines periodísticos y la Facultad de Comunicación de la Pompeu Fabra también imparte estas nociones en Fundamentos del Periodismo (6 ECTS, obligatoria), donde analiza los nuevos canales, herramientas y tendencias del periodismo. La Facultad de Comunicación de la Universidad de Castilla-La Mancha lo hace en Taller 
de Periodismo Especializado I (optativa, $4^{\circ}$ curso, 6 ECTS), mientras que la Rey Juan Carlos opta por englobarlo en Fotoperiodismo (optativa, $4^{\circ}$ curso, 6 ECTS) .

En cuanto al periodismo inmersivo y la realidad virtual está presente en 5 de los 17 centros analizados. La Facultad de Sevilla lo estudia en la asignatura optativa Periodismo multimedia y diseño gráfico digital $\left(3^{\circ}\right.$ curso, 6 ECTS $)$, mientras que Málaga lo aborda en Información en internet (optativa, $4^{\circ}$ curso, 6 ECTS) y la Facultad de Santiago de Compostela lo imparte en Narrativas periodísticas: nuevos géneros y lenguajes (obligatoria, 6 ECTS), en la que se analizan las narrativas inmersivas como experimentación en un ecosistema comunicativo en tránsito. La Facultad de Comunicación de Castilla- La Mancha lo hace en Taller de Periodismo Especializado I (optativa, $4^{\circ}$ curso, 6 ECTS) y la Autónoma de Barcelona aborda la realidad aumentada en Producción y expresión periodística en multimedia e interactivos (obligatoria, $2^{\circ}$ curso, 6 ECTS).

Cabe destacar que el Grado en Periodismo de la Universidad Autónoma de Barcelona ha estrenado este curso un nuevo plan de estudios que, poco a poco, irá sustituyendo al anterior. Este año, se estudia en $1^{\circ}$; el próximo, en $2^{\circ}$ y así sucesivamente hasta la extinción definitiva en 2025. Ante esta circunstancia, y puesto que este estudio es una foto fija del momento actual, se han analizado las guías docentes de las asignaturas que se imparten en este momento, correspondientes al nuevo plan en $1^{\circ} \mathrm{y}$ al antiguo en el resto de los cursos.

\subsection{Competencias y habilidades de los periodistas}

En los últimos años, algunas empresas periodísticas han experimentado con las tecnologías emergentes y disruptivas de la información para la narración periodística. El 31,8\% de los profesionales encuestados afirma que el medio para el que trabaja dispone de herramientas vinculadas al periodismo hi-tech, a diferencia del $68,2 \%$ restante, que sostiene que su empresa no apuesta todavía por las altas tecnologías para crear contenidos.

En cuanto a la formación que han recibido los periodistas sobre estas herramientas avanzadas, los datos indican que se ha incrementado el nivel de preparación de los periodistas, que cada vez están más necesitados de conocimientos actuales para trabajar en el complejo ecosistema mediático. El 24,8\% ha realizado algún curso, jornada, congreso o seminario formativo por cuenta propia organizado por productoras y autónomos para conocer cómo se aplica la alta tecnología al periodismo, mientras que el 75,2\% de los encuestados no cuenta con formación especializada.

Sin embargo, las competencias y habilidades adquiridas aún no se han puesto en práctica en la co-creación de contenidos, ya que el 92,7\% matiza que no utiliza técnicas avanzadas en el entorno empresarial para la elaboración colaborativa de contenidos y narrativas, aunque no desaprovechan la oportunidad de introducir 
Tabla 2.

\begin{tabular}{|c|c|c|c|c|}
\hline & Inteligencia Artificial & Drones & $\begin{array}{l}\text { Periodismo } \\
\text { Móvil }\end{array}$ & $\begin{array}{l}\text { Inmersivo / Realidad } \\
\text { virtual }\end{array}$ \\
\hline Complutense de Madrid & $x$ & $x$ & $x$ & $x$ \\
\hline Autónoma de Barcelona & $\checkmark$ & $x$ & $\checkmark$ & $\checkmark$ \\
\hline Universidad de Sevilla & $x$ & $x$ & $\checkmark$ & $\checkmark$ \\
\hline País Vasco & $x$ & $x$ & $\checkmark$ & $x$ \\
\hline Rey Juan Carlos & $x$ & $x$ & $\checkmark$ & $x$ \\
\hline Málaga & $\checkmark$ & $x$ & $\checkmark$ & $\checkmark$ \\
\hline Valladolid & $x$ & $x$ & $x$ & $x$ \\
\hline EUSA-Sevilla & $x$ & $x$ & $\checkmark$ & $x$ \\
\hline Carlos III de Madrid & $x$ & $x$ & $x$ & $x$ \\
\hline Castilla-La Mancha & $x$ & $x$ & $\checkmark$ & $\checkmark$ \\
\hline Jaume I de Castellón & $x$ & $x$ & $x$ & $x$ \\
\hline Pompeu Fabra & $x$ & $x$ & $\checkmark$ & $x$ \\
\hline Valencia & $x$ & $x$ & $x$ & $x$ \\
\hline Santiago de Compostela & $x$ & $x$ & $\checkmark$ & $\checkmark$ \\
\hline Murcia & $x$ & $x$ & $\checkmark$ & $x$ \\
\hline Zaragoza & $x$ & $x$ & $x$ & $x$ \\
\hline Rovira i Virgili & $x$ & $x$ & $x$ & $x$ \\
\hline
\end{tabular}

Fuente: Elaboración propia.

mejoras siempre que pueden. No obstante, el 71,4\% de los periodistas son conscientes de que deben adaptarse a los nuevos tiempos y aplicar estas herramientas a los métodos tradicionales de generación de noticias debido a que en un futuro habrá una cooperación entre las técnicas avanzadas y los profesionales, debido a que en el proceso de producción de la información intervienen muchos factores que ayudan a los redactores a completar el trabajo. No dudan, por tanto, de que tecnologías crearán un marco favorable para el sector periodístico.

Desde el punto de vista profesional, estos programas de última generación y tecnologías emergentes no gozan de gran aceptación. El 86,8\% cree que el uso de inteligencia artificial, drones, smartphones y realidad virtual conlleva ciertos problemas para la profesión periodística, asociados principalmente a los desafíos éticos del uso periodístico de la alta tecnología, la complejidad administrativa y la inversión 
que implica su adquisición. Por el contrario, el 13,2\% restante opina que mejora las condiciones de trabajo de los profesionales de la información al permitir a los redactores alejarse de las tareas más repetitivas y rutinarias y desarrollar otras más creativas y que aporten valor al trabajo periodístico.

Ahora bien, en relación a las ventajas para la creación de contenidos, el 47,8\% de los encuestados considera que estas tecnologías mejoran la calidad del periodismo como producto al brindar un panorama alentador para un periodismo innovador de calidad. Es decir, con la llegada de las nuevas tecnologías, las noticias se han convertido en una materia prima con la que es posible elaborar productos de mayor complejidad, con un valor añadido, gracias a la convergencia entre medios y tecnología. En el caso de los drones, por ejemplo, permiten la obtención de imágenes aéreas de difícil cobertura presencial, como las situaciones de peligro, los conflictos y los paisajes de difícil acceso, además de una mayor rapidez, movilidad y seguridad para los periodistas, mientras que la inteligencia artificial aumenta la producción de contenidos y mejora la precisión. La relativa ventaja para la narración periodística también puede conllevar mejoras en los modelos de negocio y la competitividad $(28,5 \%)$. Sin embargo, el $52,2 \%$ pone en duda los posibles beneficios que aporta la aplicación de estas herramientas, debido principalmente a la ausencia de análisis e interpretación $(77,4 \%)$.

\subsection{Propuesta de inserción curricular para el periodismo hi-tech}

Existen diferentes disciplinas en los actuales planes de estudio en las que puede englobarse la elaboración de historias periodísticas a través de las altas tecnologías (Tabla 3). En general, estas materias se reparten a lo largo de toda la titulación, aunque se concentran principalmente en $4^{\circ}$ curso y son minoritarias en el $1^{\circ}$. Mayoritariamente, son asignaturas obligatorias.

En primer lugar, destacan aquellas asignaturas con un claro componente tecnológico, centradas en el estudio de las herramientas tradicionales y emergentes que se utilizan para crear, editar y difundir información en diferentes formatos a través de los medios de comunicación. En la misma línea están las materias dedicadas al análisis de los nuevos formatos y productos para la red, enfocadas a entender los ámbitos de experimentación y producción de piezas innovadoras, que tienen que ser hipertextuales, multimedia e interactivas.

En el área de Redacción también puede englobarse el estudio del periodismo hitech, debido a que contribuye al análisis de las distintas modalidades de expresión periodística desde un punto de vista práctico. Además, su papel es crucial en la medida en que estas asignaturas están presentes en todos los planes de estudio de Periodismo. Lo mismo sucede con la Comunicación Periodística Especializada o el Periodismo Especializado, que entre sus competencias destaca la formación sobre el manejo de las tecnologías de la información y la comunicación. 
Tabla 3. Asignaturas en las que puede incluirse la enseñanza del periodismo de alta tecnología

\begin{tabular}{|c|c|c|c|c|}
\hline Modalidad & Curso & ECTS & Área temática & Competencias y habilidades de la materia \\
\hline OB & 10 & 6 & $\begin{array}{l}\text { Tecnología de la } \\
\text { Comunicación }\end{array}$ & $\begin{array}{l}\text { Herramientas tecnológicas necesarias para la } \\
\text { creación, edición y transmisión de contenidos } \\
\text { escritos y digitales. Tecnologías necesarias } \\
\text { para trabajar en los medios de comunicación, } \\
\text { teniendo en cuenta la convergencia digital. }\end{array}$ \\
\hline $\mathrm{OB}$ & $4^{\circ}$ & 6 & $\begin{array}{l}\text { Tecnologías de la } \\
\text { Gestión Periodística } \\
\text { de la Inform. Digital }\end{array}$ & $\begin{array}{l}\text { Tecnologías emergentes e incidencia en la } \\
\text { gestión periodística de la información digital. } \\
\text { Uso de Google Analytics. }\end{array}$ \\
\hline OB & $3^{\circ}$ & 6 & $\begin{array}{l}\text { Redacción } \\
\text { periodística }\end{array}$ & $\begin{array}{l}\text { Pautas para la elaboración del texto periodís- } \\
\text { tico. Tendencias y novedades informativas y } \\
\text { narrativas. }\end{array}$ \\
\hline OP & $4^{0}$ & 4 & $\begin{array}{l}\text { Periodismo e } \\
\text { Innovación } \\
\text { Tecnológica }\end{array}$ & $\begin{array}{l}\text { Herramientas de producción de documentos } \\
\text { digitales basadas en lenguajes de marcas } \\
\text { (html, xml, css). Herramientas web 2.0: blogs, } \\
\text { wikis o agregadores de contenido. }\end{array}$ \\
\hline OP & $4^{\circ}$ & 6 & $\begin{array}{l}\text { Nuevos formatos } \\
\text { y productos para } \\
\text { la red }\end{array}$ & $\begin{array}{l}\text { Innovación y desarrollo en los cibermedios } \\
\text { y su confluencia con el software. Canales, } \\
\text { formatos y productos periodísticos actuales. }\end{array}$ \\
\hline OB & $4^{0}$ & 6 & $\begin{array}{l}\text { Nuevos soportes y } \\
\text { arquitectura de la } \\
\text { información }\end{array}$ & $\begin{array}{l}\text { Producción de contenido multimedia. In- } \\
\text { fraestructura del software que sostiene las } \\
\text { plataformas de publicación digitales. Diseñar } \\
\text { sistemas eficaces de recuperación de informa- } \\
\text { ción y carga masiva de datos. }\end{array}$ \\
\hline OB & $4^{\circ}$ & 6 & $\begin{array}{l}\text { Periodismo especia- } \\
\text { lizado }\end{array}$ & $\begin{array}{l}\text { Especialización periodística en los distintos } \\
\text { géneros, narrativas, formatos, y contenidos. }\end{array}$ \\
\hline $\mathrm{OB}$ & $4^{\circ}$ & 6 & Periodismo de datos & $\begin{array}{l}\text { Herramientas para recopilar datos y presen- } \\
\text { tarlos de manera organizada. Protocolo de } \\
\text { análisis y primeros resultados }\end{array}$ \\
\hline OP & $4^{\circ}$ & 6 & $\begin{array}{l}\text { Producción periodís- } \\
\text { tica multiplataforma }\end{array}$ & $\begin{array}{l}\text { Productos periodísticos en un contexto de } \\
\text { producción periodística multiplataforma. }\end{array}$ \\
\hline $\mathrm{OB}$ & 3 & 6 & Multimedia & $\begin{array}{l}\text { Conocer las TIC para elaborar y diseñar infor- } \\
\text { mación periodística. Utilizar las tecnologías y } \\
\text { técnicas informativas en los distintos medios o } \\
\text { sistemas mediáticos combinados e interacti- } \\
\text { vos. Capacidad para comunicar en los nuevos } \\
\text { soportes digitales. }\end{array}$ \\
\hline OP & $4^{\circ}$ & 6 & $\begin{array}{l}\text { Programación y } \\
\text { Lenguajes } \\
\text { Multimedia }\end{array}$ & $\begin{array}{l}\text { Librerías, módulos, aplicaciones y otros recur- } \\
\text { sos para entornos web avanzados. Lenguajes } \\
\text { de programación y sus diversas aplicaciones } \\
\text { en entornos multimedia. }\end{array}$ \\
\hline
\end{tabular}

Fuente: Elaboración propia. 
En las materias vinculadas a la Producción Periodística también puede incluirse la enseñanza-aprendizaje de las altas tecnologías en los procesos comunicativos, pues están centradas en el estudio de las técnicas, las estrategias y las rutinas de producción de los mensajes periodísticos en los diferentes medios de comunicación y dan cabida en sus programas al conjunto de cambios que afectan a los procesos de producción de la información.

Algo similar ocurre con Multimedia, que analiza las estructuras, tipologías, contenidos y estilos de la información multimedia, así como la producción y realización de distintos contenidos conforme a una idea, utilizando las técnicas narrativas transmediales, plataformas tecnológicas y tecnologías digitales necesarias. En la misma línea se sitúa Programación y Lenguaje Multimedia, encaminada al idioma artificial diseñado para expresar procesos llevados a cabo por máquinas, como es el caso de la inteligencia artificial. Enseñan, por tanto, a crear y utilizar programas que controlen el comportamiento físico y lógico de una máquina, para expresar algoritmos con precisión, o como modo de comunicación humana.

En el ámbito de la inteligencia artificial, el big data abre nuevas posibilidades para contar historias a través de las altas tecnologías. Por eso, las asignaturas vinculadas al Periodismo de Datos suponen otra disciplina para el estudio del periodismo hitech al enseñar a buscar patrones de datos para generar automáticamente textos periodísticos.

\section{Discusión y conclusiones}

La investigación desarrollada permite dar por conseguido los objetivos planteados inicialmente de identificar la oferta formativa relacionada con el periodismo hi-tech en los grados en Periodismo de las universidades públicas españolas, conocer las competencias y habilidades que tienen los periodistas para utilizarlas en la creación de contenidos informativos y proponer diferentes áreas de conocimientos para su enseñanza-aprendizaje.

Del mismo modo, este estudio permite confirmar la primera hipótesis, ya que la enseñanza-aprendizaje de las tecnologías emergentes y disruptivas de la información empieza a identificarse en los grados en Periodismo. De los 17 planes de estudios analizados, 10 cuentan con asignaturas que dedican total o parcialmente algún contenido o módulo al estudio de esta disciplina. Contar con diferentes materias donde se inserte la enseñanza del periodismo hi-tech impulsa la innovación en las universidades, siguiendo el ritmo de los tiempos y reaccionando a la evolución de las demandas de nuevos perfiles profesionales. Por eso, los estudios de Periodismo deben seguir replanteándose, desde una perspectiva de su enfoque, sus contenidos y sus aspectos metodológicos, en línea a lo que también sugieren Casals (2006), Palomo (2013), Tejedor-Calvo y Cervi (2017), Salaverría (2016) y Schena, Besalú 
y Casellas (2018), entre otros, aunque no existe una única línea a seguir (Acosta, Costales y Rosales, 2016).

La investigación también corrobora la segunda hipótesis: los periodistas son conscientes de que deben adaptarse a los nuevos tiempos y aplicar estas herramientas a la generación y difusión de noticias. Como ejemplo, el 24,8\% de los encuestados en el marco de esta investigación ha realizado algún curso, jornada o seminario formativo por cuenta propia para conocer cómo se emplea la alta tecnología en el periodismo. Sin embargo, la carestía de algunas de estas tecnologías, así como un mercado en constante evolución, lastran su incorporación a las rutinas de la producción de información. De hecho, el 68,2\% de los periodistas afirma que el medio para el que trabaja no apuesta por las altas tecnologías para crear contenidos.

La universidad también se está adaptando poco a poco a los cambios que está sufriendo la industria periodística, aunque varios autores han señalado un distanciamiento entre las habilidades exigidas en los medios de información a los periodistas y la formación ofrecida por la academia (Du y Thornburg, 2011; Hirst, 2010; King, 2008; Mellado, 2011; Nolan, 2008; Pierce y Miller, 2007). El Nieman Lab de la Universidad de Harvard y el Informe de Predicciones del Instituto Reuters también han demandado a las instituciones académicas una importante reflexión y una amplia flexibilidad para adaptar su oferta curricular y sus metodologías a las demandas del nuevo escenario comunicativo.

En este sentido, existen diferentes áreas de conocimiento donde puede insertarse la enseñanza-aprendizaje del periodismo hi-tech. Se trata de materias que se reparten a lo largo de toda la titulación y están relacionadas con la tecnología, la redacción periodística, el periodismo especializado, la producción periodística, la programación y el lenguaje multimedia y el periodismo de datos (Hipótesis 3). Sin embargo, su estudio permite afirmar, entre otros aspectos, que todavía no existe un consenso a la hora de decidir qué materias deben encargarse de tratar los temas vinculados a las altas tecnologías en el ámbito periodístico.

La inclusión curricular del periodismo hi-tech demanda una transformación que, junto a los temarios y tipología de asignaturas, afecta igualmente a los docentes (Roblyer et al., 2010). Por un lado, se requiere una formación y reciclaje permanente del profesorado (Silva et al., 2014; Tramullas, 2016), pero, además, se necesita una reflexión continuada sobre las habilidades, las competencias y los perfiles que exige la industria. (Singh et al., 2015). Se trata, en cualquier caso, de un fenómeno global que afecta a todos los países y, por ende, al conjunto de universidades del mundo.

Paralelamente a esta exigencia de actualización curricular, Huang et. al. (2006) argumentan que, de fondo, existen unas habilidades connaturales al ejercicio del periodismo que no han sido alteradas, como el pensamiento crítico, la buena escritura, la investigación y la reflexión ética. La esencia del periodismo actual está en 
la tecnología, pero también en la calidad de los contenidos (Masip, 2016; Deuze, 2017). De ahí que López, Rodríguez y Pereira (2017) sostengan que los perfiles profesionales que perciben los periodistas en la actualidad, al margen de las herramientas concretas, cuentan con dos vectores centrales. El primero alude a los elementos básicos o fundamentos del periodismo; es decir, el conjunto de preceptos que se han sedimentado a lo largo del tiempo y forjado en procesos comunicativos a lo largo de la historia, siempre bajo un manto humanista y social, más o menos acentuado según los contextos. Y el segundo se refiere a la dimensión tecnológica, donde no se trata tanto de conocer las herramientas como de entender los fundamentos, adentrarse en los diferentes territorios y disponer de conocimientos para el trabajo individual o para el diálogo en los equipos interdisciplinares que acometen buena parte de las piezas más complejas que difunden los cibermedios actuales y que circulan por los flujos del ecosistema comunicativo actual.

En el nuevo ecosistema mediático, el gran dilema del periodismo no pasa tanto por la incorporación de las tecnologías a la práctica profesional como un conjunto de herramientas, sino por la preparación de profesionales con un perfil más tecnológico, con competencias y habilidades para aprovechar las oportunidades del modelo computacional, en el que el software ha tomado el mando (Cuesta y Camilo, 2017; Manovich, 2013) y en el que permanecen estables dimensiones que desde la óptica profesional definen la calidad periodística: relevancia, exhaustividad, diversidad, imparcialidad y precisión (Kümpel y Springer, 2015).

La cuestión estudiada no se agota en esta investigación, sino que reafirma que se trata de un debate abierto y en constante evolución. Este trabajo, a pesar de corroborar las tres hipótesis de partida, cuenta con ciertas limitaciones y deja abiertas posibilidades para futuros estudios. Una muestra más amplia de planes de estudio y de periodistas encuestados habría posibilitado la extracción de más condiciones extrapolables a la totalidad del mercado, de la misma forma que la inclusión de planes de estudio de ámbito internacional ayudaría a comparar el desarrollo de estas iniciativas con la de otros países.

\section{Referencias}

Acosta Damas, Maribel; Costales Pérez, Zenaida y Rosales Vicente, Beatriz (2016). Formación por competencias profesionales en la carrera de Periodismo. En: Revista Cubana de Educación Superior, vol.35, n ${ }^{0} 1$. La Habana: Ministerio de Educación Superior, 75-84.

Aguado, Guadalupe (2019). Repercusión en el ejercicio del periodismo de la regulación del uso de drones en Europa. En Flores Vivar, Jesús (coord.). En: Tecnologías del ecosistema periodístico. Realidad inmersiva, drones y otras tecnologías disruptivas en la nueva ecología de los medios. Salamanca: Comunicación Social. 
Anderson, C.W. (2012). Notas hacia un análisis del periodismo computacional. En: HIIG Discussion Paper Series. Berlin: HIIG. https://ssrn.com/abstract=2009292

Bardin, Laurence (1977). Análise de conteúdo. Lisboa: Edições 70.

Barrat, James (2013). Our final invention: Artificial intelligence and the end of the human era. Nueva York: Thomas Dunne Books. St. Martin Press.

Barreno Cortés, Jorge (2019). La miniaturización de los drones y sus múltiples usos periodísticos. En Flores Vivar, Jesús (coord.). Tecnologías del ecosistema periodístico. Realidad inmersiva, drones y otras tecnologías disruptivas en la nueva ecología de los medios. Salamanca: Comunicación Social.

Bermejo Casado, Irene; Campos García de Quevedo, Gloria y Sánchez-Bayón, Antonio (2017). Estrés y síndrome de desgaste profesional en la organización de eventos en el sector de las agencias de comunicación. En Revista Latina de Comunicación Social, $\mathrm{n}^{0} 72$. La Laguna: Sociedad Latina para la Comunicación Social, 1.394-1.417.

Bunz, Mercedes (2010). In the US, algorithms are already reporting the news. The Guardian. Consultado el 24 de junio de 2020 en https://cutt.ly/FtT3IoL

Caminero Fernández, Lidia y Sánchez-García, Pilar (2018). El perfil y formación del ciberperiodista en redacciones nativas digitales. En: Hipertext.net, $\mathrm{n}^{0} 16$. Barcelona: Universitat Pompeu Fabra, 4-15. doi: http://doi.org/10.31009/hipertext. net.2018.i16.04

Canavilhas, João (2015). Nuevos medios, nuevo ecosistema. En: El profesional de la información, vol. 24, $n^{\circ} 4$. Barcelona: EPI, 357-362. doi: http://dx.doi.org/10.3145/ epi.2015.jul.01

Cantero de Julián, Juan Ignacio; Calvo Rubio, Luis Mauricio y Benedicto Solsona, Miguel Ángel (2020). La tenue apuesta por los vídeos en $360^{\circ}$ en las estrategias transmedia de las televisiones autonómicas españolas. En: Revista Latina de Comunicación Social, $\mathrm{n}^{0} 75$. La Laguna: Sociedad Latina para la Comunicación Social, 415-433.

Casals, María Jesús (2006). La enseñanza del periodismo y las nuevas tecnologías de la información y de la comunicación. En: Estudios sobre el mensaje periodístico, $\mathrm{n}^{0}$ 12. Madrid: Universidad Complutense de Madrid, 59-70.

Casero-Ripollés, Andreu (2012). Beyond newspapers: News consumption among young people in the digital era. En: Comunicar, vol.20, $\mathrm{n}^{\circ} 39$. Huelva: Grupo Comunicar, 151-158. doi: https://doi.org/10.3916/C39-2012-03-05

Christensen, Clayton M. (1999). El dilema de los innovadores. Buenos Aires: Ediciones Granica SA. 
Codina, Lluís (2017). Revisiones sistematizadas y cómo llevarlas a cabo con garantías: Systematic reviews y SALSA Framework. Consultado el 24 de junio de 2020 en https://www.lluiscodina.com/revision-sistematica-salsa-framework

Cuesta, Óscar Julián y Chacón, Juan Camilo (2017). Evaluación de la formación de periodistas: entre las destrezas tecnológicas, las habilidades investigativas y el pensamiento crítico. En: Escenarios, vol.15, $\mathrm{n}^{0} 1$. Bogotá: Universidad Autónoma de El Caribe, 25-35. doi: http://dx.doi.org/10.15665/esc.v15i1.1119

De la Peña, Nonny; Weil, Peggy; Llobera, Joan; Giannopoulos, Elias; Pomés, Ausiàs; Spaniang, Bernhard; Friedman, Doron; Sánchez-Vives, María V. y Slater, Mel (2010). Immersive journalism: Immersive virtual reality for the first-person experience of news. En: Presence: Teleoperators and virtual environments, vol.19, $\mathrm{n}^{\circ}$ 4. Cambridge: MIT Press, 291-301. doi: http://dx.doi.org/10.1162/pres_a_00005

Deuze, Mark (2017). Considering a possible future for Digital Journalism. En: Revista Mediterránea de Comunicación, vol.8, $\mathrm{n}^{0} 1$. Alicante: Universidad de Alicante, 9-18. doi: https://doi.org/10.14198/MEDCOM2017.8.1.1

Domínguez Martín, Eva (2015). Periodismo inmersivo o cómo la realidad virtual y el videojuego influyen en la interfaz e interactividad del relato de actualidad. En: El profesional de la información, vol.24, $\mathrm{n}^{\circ} 4$. Barcelona: EPI, 413-423. doi: http:// dx.doi.org/10.3145/epi.2015.jul.08

Du, Ying Roselyn y Thornburg, Ryan (2011). The Gap Between Online Journalism Education and Practice. The Twin Surveys. En: Journalism and Mass Communication Educator. Thousand Oaks: Sage, 218-230.

Eudes, Yves (2014). The journalists who never sleep. The Guardian. Consultado el 24 de junio de 2020 en https://cutt.ly/5rY9c45

Fernández Barrero, María Ángeles (2018a). El periodismo que vuela. Drones, 3D, Smartphones y Robots, Tecnologías Emergentes para la Profesión Periodística. Sevilla: Fénix Editora.

Fernández Barrero, María Ángeles (2018b). Periodismo y drones. Retos y oportunidades del uso de drones para la narración informativa en España. En: Doxa Comunicación, 26, 35-58.

Fidler, Roger (1997). Mediamorphosis. Understanding new media. Thousand Oaks: Pine Forge Press.

Flores Vivar, Jesús Miguel (2019). Tecnologías del ecosistema periodístico. Realidad inmersiva, drones y otras tecnologías disruptivas en la nueva ecología de medios. Salamanca: Comunicación Social.

Gaitán Moya, Juan Antonio y Piñuel Raigada, José Luis (1998). Técnicas de investigación en comunicación social: elaboración y registro de datos. Madrid: Síntesis. 
García-Avilés, José Alberto (2018). Resultados de la innovación en los laboratorios de medios: el caso de El confidencial.LAB. En: El profesional de la información, vol.27, nº2. Barcelona: EPI, 359-366. doi:https://doi.org/10.3145/epi.2018.mar.14 Gómez-Calderón, Bernardo José; Roses, Sergio y García-Borrego, M. (2017). En: Revista Mediterránea de Comunicación, vol.8, $\mathrm{n}^{0} 1$. Alicante: Universidad de Alicante, 191-200. doi: https://doi.org/10.14198/MEDCOM2017.8.1.14

Gómez Diago, G. (2010). Triangulación metodológica: paradigma para investigar desde la ciencia de la comunicación. En: Razón y palabra, vol.15, nº 72, México: ITESM Campus Estado de México.

Hermida, Alfred y Thurman, Neil (2008). A clash of cultures. The integration of user-generated content within professional journalistic frameworks at British newspaper websites. En: Journalism practice, vol.2, nº3. Londres: Routledge, 343-356. doi: https://doi.org/10.1080/17512780802054538,

Herranz de la Casa, José María; Sidorenko Bautista, Pavel y Cantero de Julián, Juan Ignacio (2019). Realidad virtual y periodismo inmersivo: nuevas formas de contar historias para los periodistas. En: Ufarte Ruiz, María Jose (ed.). Nuevos perfiles profesionales para el mercado periodístico. Salamanca: Comunicación Social Ediciones y Publicaciones.

Herring, Susan (2010). Web content analysis: Expanding the paradigm. En: Hunsinger, Jeremy; Klastrup, Lisbeth; Allen, Matthew M. (eds.). International handbook of internet research. Nueva York: Springer Verlag.

Hewett, Jonathan (2015). Learning to teach data journalism: Innovation, influence and constraints. En: Journalism, vol.17, nº1. Londres: SAGE, 119-137.

Hirst, Martin (2010). Journalism Education “Down Under”. A Tale of Two Paradigms. En: Journalism Studies, vol.11, nº1. Nueva York: Routledge, 83-98.

Huang, Edgar; Davison, Karen; Shreve, Stephanie; Davis, Twila; Bettendorf, Elizabeth y Nair, Anita (2006). Bridging Newsrooms and Classrooms. Preparing the Next Generation of Journalists for Converged Media. En: Journalism and Communication Monographs, $\mathrm{n}^{0} 262$. Columbia: Association for Education in Journalism and Mass Communication.

King, Elliot (2008). The Role of Journalism History, and the Academy, in the Development of Core Knowledge in Journalism Education. En: Journalism \& Mass Communication Educator, vol.63, nº2. Thousand Oaks: Sage, 166-178.

Krippendorf, Klaus (2004). Content analysis: An introduction to its methodology. Thousand Oaks: Sage.

Kümpel, Anna Sophie y Springer, Nina (2015). Commenting Quality: Effects of User Comments on Perceptions of Journalistic Quality. En: The Future of Journalism, vol.5, no 3, 353-366 doi: https://doi.org/10.5771/2192-4007-2016-3-353. 
Larrondo Ureta, Ainara y Teixeira, Juliana Fernandes (2016). La convergencia narrativa en el periodismo móvil. Aproximación a la integración del contenido audiovisual en los productos nativos para iPad. En: Estudios sobre el Mensaje Periodístico, vol.22, nº2. Madrid: Universidad Complutense de Madrid, 777-792. Lecompte, Celeste (2015). Automation in the Newsroom. How algorithms are helping reporters expand coverage, engage audiences, and respond to breaking news. Consultado el 24 de junio de 2020 en https://cutt.ly/OtTXdgS

López García, Xosé; Rodríguez Vázquez, Ana Isabel y Pereira-Fariña, Xosé (2017). Competencias tecnológicas y nuevos perfiles profesionales: desafíos del periodismo actual. En: Comunicar, vol. XXV, $n^{0}$ 53. Huelva: Grupo Comunicar, 81-90. doi: https://doi.org/10.3916/C53-2017-08

López García, Xosé; Silva Rodríguez, Alba; Vizoso García, Antonio; Westlund, Óscar; Canavilhas, Joao (2019). Mobile journalism: Systematic literature review. En: Comunicar, vol. 2, $\mathrm{n}^{\circ} 1$. Huelva: Grupo Comunicar 9-18. doi: https://doi. org/10.3916/C59-2019-01

López Hidalgo, Antonio (2016). El periodismo que contará el futuro. En: Revista Chasqui, $\mathrm{n}^{0} 131$. Quito: CIESPAL, 239-256.

López Hidalgo, Antonio y Aramburú Moncada, Luisa Gabriela (2016). La apuesta de las universidades por el periodismo dron. En: TecCom Studies: Estudios de Tecnología y Comunicación, $n^{\circ}$ 7. Madrid: Internet Media Lab, 123-134.

López Hidalgo, Antonio; Ufarte Ruiz, María José (2016). Laboratorios de periodismo en España. Nuevas narrativas y retos de futuro. En: Ámbitos: revista internacional de comunicación, 34 .

Lozano, Vicente (2017). La comunicación líquida de Bauman. El Mundo. Consultado el 24 de junio de 2020 en https://cutt.ly/mtEy8Mj

Manovich, Lev (2013). Software Takes Command. Nueva York: Bloomsbury Academic.

Masip, Pere (2016). Investigar el periodismo desde la perspectiva de las audiencias. En: El Profesional de la Información, 25(3). Barcelona: EPI, 323-330. https://doi. org/10.3145/epi.2016.may.01

McLuhan, Marshall (1964). Media ecology. A first look at communication theory, 312-322.

Mellado, Claudia (2011). Examining Professional and Academic Culture in Chilean. Journalism and Mass Communication Education. En: Journalism Studies, vol.12, $\mathrm{n}^{\circ}$ 3. Nueva York: Routledge, 375-391.

Micó Sanz, Josep Lluís y Pérez Soler, Susana (2015). La participación en los medios de comunicación a través de Twitter. Estudio comparativo entre los periodistas 
catalanes y belgas. En: Sur le journalisme, vol.4, $\mathrm{n}^{0} 2$. Bruselas: Universite Libre de Bruxelles, 62-73.

Moreiro González, José Antonio; Azcárate Aguilar Amat, Pilar; Marzal GarcíaQuismondo, Miguel Ángel; Tejada Artigas, Carlos Miguel y Vergueiro, Waldomiro (2008). Desarrollo profesional y opinión sobre la formación recibida de los titulados universitarios en información y documentación de las universidades públicas de Madrid (2000-2005). En: El profesional de la información, vol.17, nº3. Barcelona: EPI, 261-272.

Murcia Verdú, Francisco José y Ufarte Ruiz, María José (2019). Mapa de riesgos del periodismo hi-tech. En: Hipertext.net, $\mathrm{n}^{0} 18$. Barcelona: Universitat Pompeu Fabra, 47-55. doi: 10.31009/hipertext.net.2019.i18.05

Newman, Nic (2020). Journalism, media, and technology trends and predictions 2020. Oxford: Reuters Institute for the Study of Journalism.

Nolan, David (2008). Journalism, Education and the Formation of 'Public Subjects'. En: Journalism, nº9. Londres: SAGE, 733-949.

Okuda Benavides, Mayumi y Gómez Restrepo, Carlos (2005). Metodología de investigación y lectura crítica de estudios. Métodos de investigación cualitativa: triangulación. En: Revista colombiana de psiquiatría, vol.34, nº1. Bogotá: Asociación Colombiana de Psiquiatría.

Palomo, Bella (2013). Claves de la implantación y la expansión del perfil social del periodista. En: Comunicación y Medios, $\mathrm{n}^{\circ} 28$. Santiago de Chile: Universidad de Chile, 113-129.

Paulussen, Steve (2016). Innovation in newsrooms. En Witschge, Tamara; Anderson, C.W.; Domingo, David y Hermida, Alfred (Eds.), The SAGE Handbook of Digital Journalism, Londres: Sage.

Pavlik, John V. (2001). Journalism and new media. Nueva York: Columbia University Press

Perlado Lamo de Espinosa, Marta, Papí Gálvez, Natalia y Bergaz Portolés, María (2019). Del planificador de medios al experto en medios: El efecto digital en la publicidad. En: Comunicar, vol. 27, no 59. Huelva: Grupo Comunicar, 105-114. doi: https://doi.org/10.3916/C59-2019-10

Pierce, Tamyra y Miller, Tommy (2007). Basic Journalism Skills Remain Important in Hiring. En: Newspaper Research Journal, vol.28, nº4. Londres: Sage, 51-61.

Postman, Neil (1970). The humanism of media ecology. In Proceedings of the Media Ecology Association, vol. 1, no 1. Orono: Media Ecology Association, 10-16.

Roblyer, Margaret D.; McDaniel, Michel; Webb, Marsena; Herman, James y Witty, James Vince (2010). Findings on Facebook in higher education: A comparison of 
college faculty and student uses and perceptions of social networking sites. En: The Internet and higher education, vol.13, $\mathrm{n}^{\circ} 3$. Nueva York: Pergamon, 134-140. Salaverría, Ramón (2014). Periodismo en 2014: balance y tendencia. En: Cuaderno de Periodistas, $\mathrm{n}^{0}$ 29. Madrid: APM, 9-22.

Salaverría, Ramón (2016). Redefinir al comunicador. En: El profesional de la información 25(2). Barcelona: EPI, 163-167. https://doi.org/10.3145/epi.2016.mar.02 Salaverría, Ramón (2019). Transmedia production: Key steps in creating a storyworld. En: Deuze, Mark y Prenger, Mirjam (eds.). Making media. Production, practicees, and professions. Ámsterdam: Amsterdam University Press, 453-461.

Salaverría, Ramón; García Avilés, José Alberto y Masip, Pere (2007). Convergencia periodística: propuesta de definición teórica y operativa. En Documento de trabajo, Proyecto Convergencia digital en los medios de comunicación. Consultado el 24 de junio de 2020 en http://www.ae-ic.org/santiago2008/contents/pdf/ comunicaciones/134.pdf

Schena, Jucinara; Besalú, Reinald y Singla-Casellas, Carles (2018). Valoraciones actualizadas de las competencias profesionales en la práctica laboral de los periodistas españoles. En: Revista Latina de Comunicación Social, $\mathrm{n}^{0} 73$. La Laguna: Sociedad Latina para la Comunicación Social, 531-555. doi: http://doi.org/10.4185/ RLCS-2018-1268

Sánchez, Pilar (2013). Propuesta metodológica para el análisis comparado de los planes de estudio en la licenciatura y el grado de periodismo en la universidad española. En: Actas del $2^{\circ}$ Congreso Nacional sobre Metodología de la Investigación en Comunicación, 365-382.

Singh, Jagtar; Grizzle, Alton; Joan, Sin Yee y Hope Culver, Sherri (Eds.) (2015). Media and Information Literacy for the Sustainable Development Goals. Goteborg: Nordicom.

Silva, Bento; Araújo, Alexandra; Vendramini, Claudette; Martins, Ronei, Piovezan, Nayane; Prates, Eli y Joly, María Cristina (2014). Aplicação e uso de tecnologias digitais pelos professores do ensino superior no Brasil e em Portugal. En: Educação, Formação \& Tecnologias, vol.7, $\mathrm{n}^{\circ} 1$. Lisboa: EDUCOM, 3-18.

Soler Pujals, Pere y Enrique Jiménez, Ana María (2012). Reflexión sobre el rigor científico en la investigación cualitativa. En: Estudios sobre el mensaje periodístico, vol.18. Madrid: Universidad Complutense de Madrid, 879-888. doi: https://doi. org/10.5209/rev_ESMP.2012.v18.40966

Tejedor-Calvo, Santiago y Cervi, Laura (2017). Análisis de los estudios de Periodismo y Comunicación en las principales universidades del mundo. Competencias, objetivos y asignaturas. En: Revista Latina de Comunicación Social, $n^{0} 72$. La La- 
guna: Sociedad Latina para la Comunicación Social, 1.626-1.647. doi: https://doi. org/10.4185/RLCS-2017-1238

Tejedor-Calvo, Santiago; Corpus, Roberto-Silva; Lozano, Fernando (2011). La formación del ciberperiodista 2.0. En: Quadros, Cláudia; Caetano, Kati; Laranjeira, Álvaro. Jornalismo e convergência: ensino e práticas profissionais. Covilhã: Livros Labcom, 21-42.

Tramullas, Jesús (2016). Hannibal ad portas, o los futuros perfiles profesionales de la información. En: El profesional de la información 25(2). Barcelona: EPI, 157-162. doi: http://dx.doi.org/10.3145/epi.2016.mar.01

Túñez López, José Miguel; Toural Bran, Carlos y Cacheiro Requeijo, Santiago (2018). Uso de bots y algoritmos para automatizar la redacción de noticias: percepción y actitudes de los periodistas en España. En: El profesional de la información, vol.27, n ${ }^{\circ}$ 4. Barcelona: EPI, 750-758. doi: https://doi.org/10.3145/epi.2018.jul.04 Ufarte Ruiz, María José (2019). Nuevos perfiles profesionales para el mercado periodístico. Salamanca: Comunicación Social.

Ufarte Ruiz, María José; Manfredi Sánchez, Juan Luis (2019). Algoritmos y bots aplicados al periodismo. El caso de Narrativa Inteligencia Artificial: estructura, producción y calidad informativa. En: Doxa Comunicación, nº29. Madrid: CEU Ediciones, 213-233. doi: https://doi.org/10.31921/doxacom.n29a11

Van Der Haak, Bregtje; Parks, Michael y Castells, Manuel (2012). The Future of Journalism: Networked Journalism. En: International Journal of Communications, $\mathrm{n}^{\circ}$ 6. Salem: NAUN, 2.923-2.938.

Villani, Cédric (2018). For Meaningful Artificial Intelligence. Towards a French and European Strategy. Consultado el 24 de junio de 2020 en https://cutt.ly/wtEaz9v Villareal Larrinaga, Oskar y Landeta Rodríguez, Jon (2010). El estudio de caso como metodología de investigación científica en dirección y economía de la empresa. Una aplicación a la internacionalización. En: Investigaciones europeas de dirección y economía de la empresa, vol.16, $\mathrm{n}^{\circ} 3$. Madrid: AEDEM, 31-52. doi: https://doi.org/10.1016/S1135-2523(12)60033-1

Westlund, Oscar (2013). Mobile news: A review and model of journalism in an age of mobile media. En: Digital journalism, vol.1, nº1. Londres: T\&F Informa, 6-26. doi: http://dx.doi.org/10.1080/21670811.2012.740273

Yin, Robert K. (1981). The case study crisis: Some answers. En: Administrative science quarterly, vol.26, $\mathrm{n}^{0} 1$. Londres: SAGE, 58-65. 
